*Supporting information

\title{
Effect of Aging in Nitrogen and Air on the Properties of Biocrude produced by Hydrothermal Liquefaction of Spirulina
}

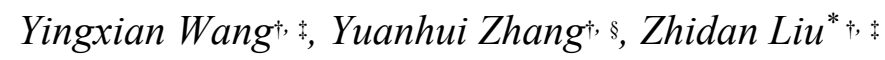

${ }^{\dagger}$ Laboratory of Environment-Enhancing Energy (E2E), College of Water Resources and Civil Engineering, China Agricultural University, Qinghua Donglu 17, Beijing 100083, China

$\$$ Key Laboratory of Agricultural Engineering in Structure and Environment, Ministry of Agriculture, Qinghua Donglu 17, Beijing 100083, China

$\S$ Department of Agricultural and Biological Engineering, University of Illinois at Urbana-Champaign, Urbana, IL 61801, USA

*Corresponding author.

Fax: +86-10-62737329; Tel.: +86-10-62737329.

Email: zdliu@cau.edu.cn (Z. Liu) 


\section{Table S1}

Changes in mass of biocrude oil at different aging times and conditions.

\begin{tabular}{ccccc}
\hline & \multicolumn{4}{c}{ Mass changes at different aging times a $(\%)$} \\
\cline { 2 - 5 } Biocrude oil & 2 weeks & 4 weeks & 8 weeks & 12 weeks \\
\hline Air-15 & 0.48 & 1.61 & 2.54 & 3.69 \\
Air-35 & 0.73 & 3.33 & 5.40 & 7.21 \\
$\mathrm{~N}_{2}-15$ & 0.15 & 0.42 & 0.55 & 0.77 \\
$\mathrm{~N}_{2}-35$ & 0.35 & 0.71 & 1.04 & 1.39 \\
\hline
\end{tabular}

${ }^{a}$ Biocrude oil was weighted each time after measurement and before storage. Every mass change was based on the original mass of biocrude oil. 


\section{Table S2}

Organic compounds of fresh and aged biocrude oils stored at different conditions through GC-MS analysis.

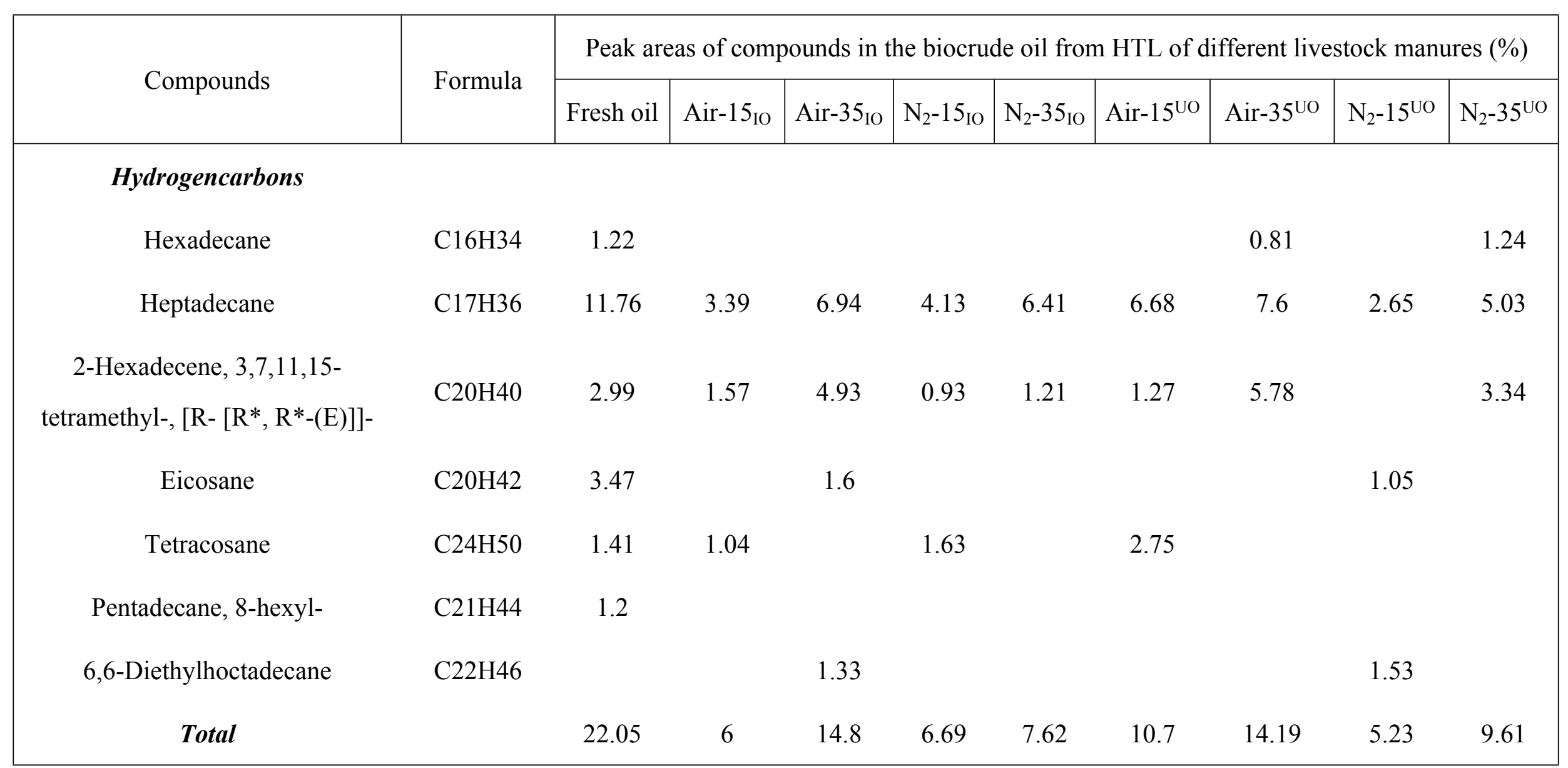




\begin{tabular}{|c|c|c|c|c|c|c|c|c|c|c|}
\hline Esters & & & & & & & & & & \\
\hline Dimethyl phthalate & $\mathrm{C} 10 \mathrm{H} 10 \mathrm{O} 4$ & 3.41 & & & & & & & & \\
\hline Acetic acid n-octadecyl ester & $\mathrm{C} 20 \mathrm{H} 40 \mathrm{O} 2$ & 2.87 & & & 1.96 & 1.94 & 3.52 & & 3.81 & 1.23 \\
\hline Di-n-octyl phthalate & $\mathrm{C} 24 \mathrm{H} 38 \mathrm{O} 4$ & & 1.83 & 1.68 & & 1.26 & 1.37 & 0.89 & 1.58 & \\
\hline Tetracosyl acetate & $\mathrm{C} 26 \mathrm{H} 52 \mathrm{O} 2$ & & & 2.09 & & & & & & \\
\hline Hexadecanoic acid, methyl ester & $\mathrm{C} 17 \mathrm{H} 34 \mathrm{O} 2$ & & & & 3.25 & 2.03 & & 2.5 & 1.05 & 2.11 \\
\hline Cyclopropaneoctanoic acid, 2-[[2- & & & & & & & & & & \\
\hline [(2-ethylcyclopropyl) methyl] & $\mathrm{C} 22 \mathrm{H} 38 \mathrm{O} 2$ & & & & 0.76 & & & & & \\
\hline cyclopropyl] methyl]-, methyl ester & & & & & & & & & & \\
\hline 1-Hexadecanol, acetate & $\mathrm{C} 18 \mathrm{H} 36 \mathrm{O} 2$ & & & & 0.33 & & & & & \\
\hline
\end{tabular}


6,9,12-Octadecatrienoic acid,

$$
\text { methyl ester }
$$

Malonic acid, 2-methylpentyl nonyl

$$
\text { ester }
$$

Phenylacetic acid, 2-ethylhexyl

$$
\text { ester }
$$

2,6-Nonadienoic acid, 9-(3,3-

dimethyloxiranyl)-3,7-dimethyl-, $\quad \mathrm{C} 16 \mathrm{H} 26 \mathrm{O} 3$

methyl ester, (E, E)-

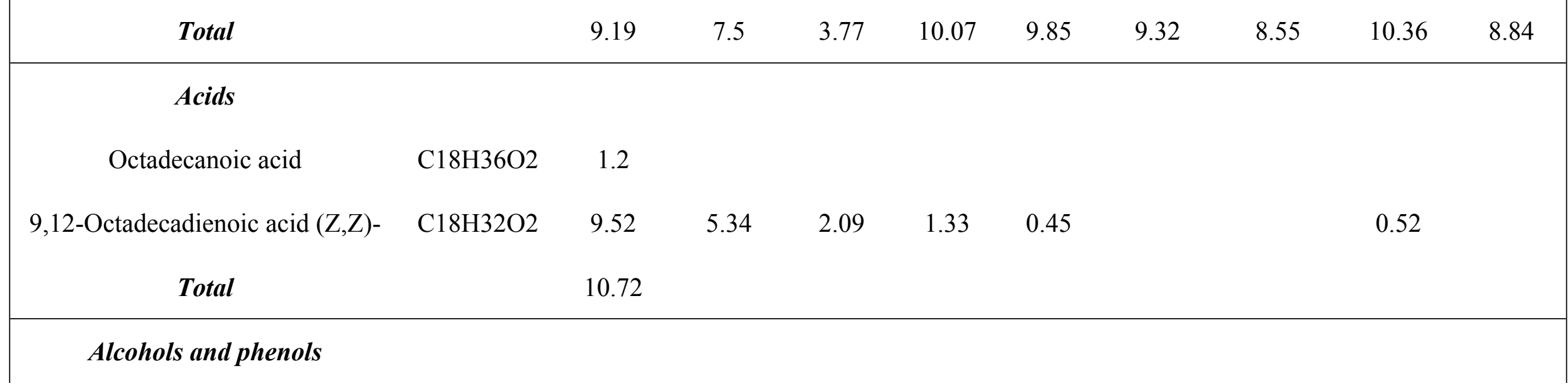




\begin{tabular}{|c|c|c|c|c|c|c|c|c|c|c|}
\hline Phenol & C6H6O & 2.92 & & & & & & & & \\
\hline 2-Hexyl-1-octanol & $\mathrm{C} 14 \mathrm{H} 30 \mathrm{O}$ & & & & & & & & & 1.25 \\
\hline .gamma.-Sitosterol & $\mathrm{C} 29 \mathrm{H} 50 \mathrm{O}$ & & & & & & & & 1.29 & \\
\hline 1-Heptadec-1-ynyl-cyclopentanol & $\mathrm{C} 22 \mathrm{H} 40 \mathrm{O}$ & & & & & & & 0.64 & & \\
\hline Phenol, 2,4-bis(1,1-dimethylethyl)- & $\mathrm{C} 14 \mathrm{H} 22 \mathrm{O}$ & 2.22 & & & & & & & & \\
\hline $\begin{array}{c}\text { Phenol, 2,2'-methylenebis[6-(1,1- } \\
\text { dimethylethyl)-4-methyl- }\end{array}$ & $\mathrm{C} 23 \mathrm{H} 32 \mathrm{O} 2$ & 18.87 & 43.47 & 43.14 & 45.12 & 34.28 & 23.88 & 25.3 & 56.02 & 50.12 \\
\hline Phenol, 2,4-bis(1-phenylethyl)- & $\mathrm{C} 22 \mathrm{H} 22 \mathrm{O}$ & & & & & & 1.38 & & & \\
\hline Total & & 25.18 & 43.47 & 43.14 & 45.12 & 34.28 & 25.26 & 25.94 & 57.31 & 51.37 \\
\hline \multicolumn{11}{|l|}{ Ketones } \\
\hline 7-Ethyl-4,6-heptadecandione & $\mathrm{C} 19 \mathrm{H} 36 \mathrm{O} 2$ & 5.73 & & 3.38 & & & & & & \\
\hline Oxybenzone & $\mathrm{C} 14 \mathrm{H} 12 \mathrm{O} 3$ & & 1.67 & & 2.9 & & & & & 2.19 \\
\hline Octabenzone & $\mathrm{C} 21 \mathrm{H} 26 \mathrm{O} 3$ & & 5.45 & & 6.61 & 3.21 & & & 8.36 & 4.83 \\
\hline
\end{tabular}


7-Hydroxy-6,9a-dimethyl-3-

methylene-decahydro-azuleno[4,5- $\mathrm{C} 15 \mathrm{H} 20 \mathrm{O} 4$

b] furan-2,9-dione

\section{Total}

5.73

7.12

3.38

9.51

3.21

1.84

0

7.02

\section{Nitrogen-containing compounds}

Quinoline, 1,2-dihydro-2,2,4-

trimethyl-

C12H15N

1.05

1-(3-Methylbutyryl) pyrrolidine

C9H17NO

2.1

$3.13 \quad 3.52$

1,4-Bis(3-aminopropyl) piperazine $\quad \mathrm{C} 10 \mathrm{H} 24 \mathrm{~N} 4$

1.05

2,5-Piperazinedione, 3,6-bis(2-

$\mathrm{C} 12 \mathrm{H} 22 \mathrm{~N} 2 \mathrm{O}$

methylpropyl)-

2

Pyrrolo[1,2-a] pyrazine-1,4-dione, C11H18N2O

hexahydro-3-(2-methylpropyl)-

2

Tetradecanamide

C14H29NO 4.24

N-Methyldodecanamide

$\mathrm{C} 13 \mathrm{H} 27 \mathrm{NO} \quad 2.83$ 


\begin{tabular}{|c|c|c|c|c|c|c|c|c|c|c|}
\hline N, N-Dimethyldodecanamide & C14H29NO & 2.17 & 2.99 & 2.22 & 4.82 & 2.8 & 3.15 & 3.01 & 3.79 & 3.62 \\
\hline Octadecanamide, N-butyl- & $\mathrm{C} 22 \mathrm{H} 45 \mathrm{NO}$ & 1.64 & 3.48 & 4.92 & 2.49 & 4.52 & 5.28 & 5.45 & 2.28 & 2.89 \\
\hline N-Decanoylmorpholine & $\mathrm{C} 14 \mathrm{H} 27 \mathrm{NO} 2$ & 8.12 & 5.99 & 8.91 & 4.96 & 9.64 & 10.97 & 11.79 & 3.25 & 5.79 \\
\hline Octadecanamide & $\mathrm{C} 18 \mathrm{H} 37 \mathrm{NO}$ & & 5.24 & & 3.82 & 5.13 & 6.09 & 6.41 & & 1.5 \\
\hline 9-Octadecenamide, (Z)- & C18H35NO & & 3.94 & 3.08 & 5.15 & 5.7 & 6.93 & 3.97 & 3.23 & 2.52 \\
\hline Dodecanamide, N, N-diethyl- & $\mathrm{C} 16 \mathrm{H} 33 \mathrm{NO}$ & & 2.17 & & & & & & & \\
\hline n-Dodecanoylpyrrolidine & $\mathrm{C} 16 \mathrm{H} 31 \mathrm{NO}$ & & 1.52 & & & & & & & 1.8 \\
\hline $\begin{array}{l}\text { Pyrrolidine, 1-(6-methyl-1- } \\
\text { oxooctadecyl)- }\end{array}$ & $\mathrm{C} 23 \mathrm{H} 45 \mathrm{NO}$ & & 2.52 & 3.6 & & & 3.21 & 3.64 & 2.86 & \\
\hline $\begin{array}{l}\text { Oxalic acid, monoamide, N-(2- } \\
\text { phenylethyl)-, isohexyl ester }\end{array}$ & $\mathrm{C} 16 \mathrm{H} 23 \mathrm{NO} 3$ & & & 2.07 & & 1.13 & 1.7 & & & \\
\hline 8-Methyl-6-nonenamide & $\mathrm{C} 10 \mathrm{H} 19 \mathrm{NO}$ & & & & & 1.36 & & & & \\
\hline Pyrrolidine, 1-(1-oxooctadecyl)- & $\mathrm{C} 22 \mathrm{H} 43 \mathrm{NO}$ & & & 2.67 & 2 & 2.59 & & & & \\
\hline Oleic diethanolamide & $\mathrm{C} 22 \mathrm{H} 43 \mathrm{NO} 3$ & & & 1.42 & & 1.72 & 2.03 & 2.47 & & 1.5 \\
\hline
\end{tabular}


Tricyclo[4.3.0.0(3,8)]nonan-2-ol, 2-

(aminomethyl)-, stereoisomer

$\mathrm{C} 10 \mathrm{H} 17 \mathrm{NO}$

1.54

.beta.-L-Lyxo-Hexopyranose, 3-

(acetylamino)-2,3,6-trideoxy-, 1,4- C12H19NO6

1.03

\section{diacetate}

9-Octadecenamide, N-(1-

methylethyl)-

$\mathrm{C} 21 \mathrm{H} 41 \mathrm{NO}$

0.64

n-Octyl guanidine

C9H21N3

1.19

13-Docosenamide, (Z)- $\quad \mathrm{C} 22 \mathrm{H} 43 \mathrm{NO}$

9-Octadecenamide, N, N-dimethyl- C20H39NO

Nonadecanamide

C19H39NO

1.07

Total

27.13

30.57

32.82

27.28

44.59

52.88

51.32

18.22

23.16 
Figure S1. 3D Van Krevelen diagram of fresh biocrude oil and aged biocrude oils stored at different conditions. UO stands for the "upper oil" in the top of biocrude oil, and IO stands for the "inner oil" under UO.

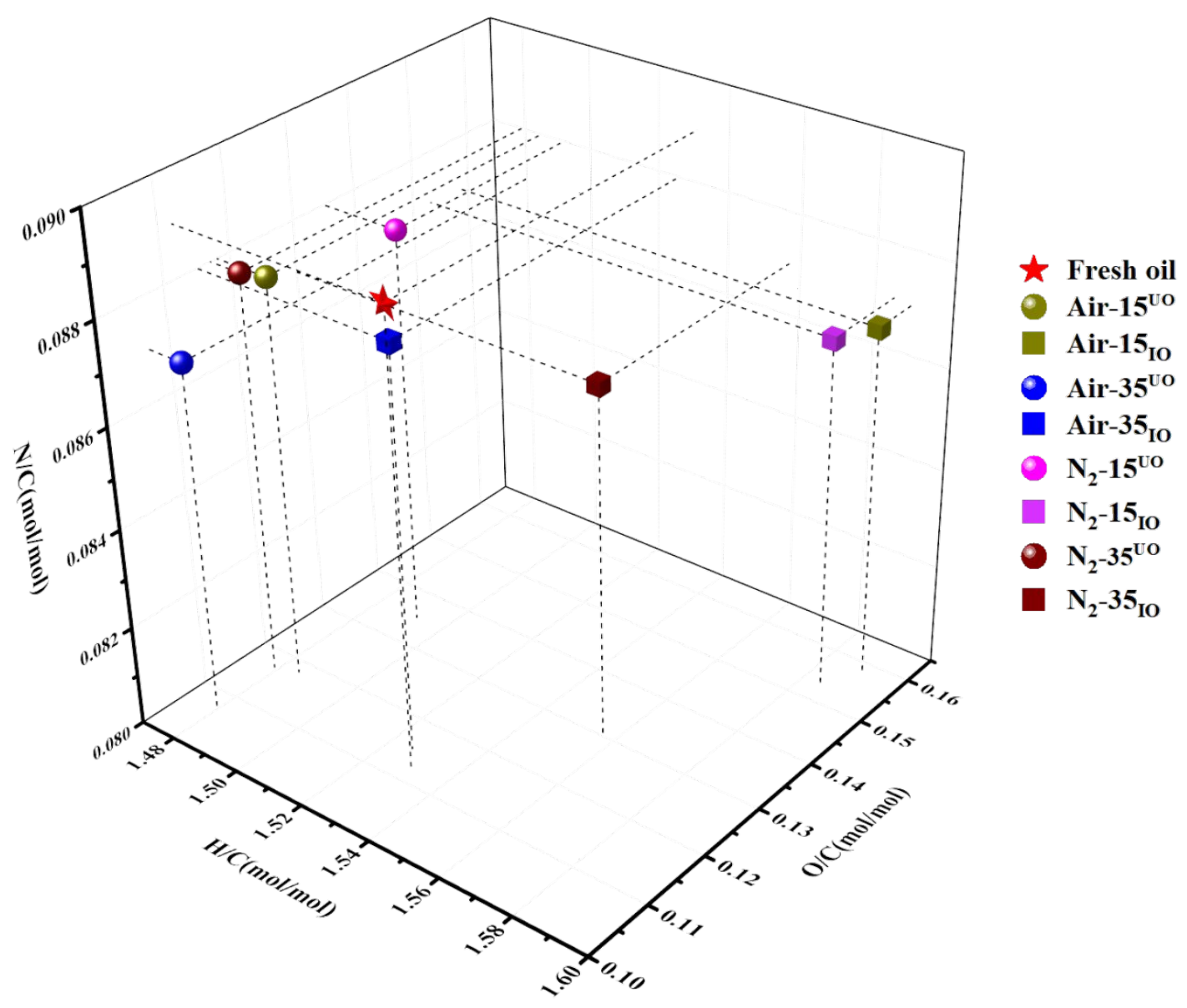

